\title{
VIVERE E MORIRE IN UN CENTRO DI PELLEGRINAGGIO LONGOBARDO: SAN MICHELE DI OLEVANO SUL TUSCIANO (SECC. VIII-IX)
}

\section{ALESSANDRO DI MURO}

UDC: $27-523(450.728) " 07 / 08 "$

Preliminary communication

Manuscript received: 29. 10. 2016.

Revised manuscript accepted: 17. 02. 2017.

DOI: 10.1484/J.HAM.5.113731
A. Di Muro

Dipartimento di Scienze umane Università degli Studi della Basilicata

Via Nazario Sauro, 85

85100 Potenza, Italia

alessandro.dimuro@unibas.it | alexdimuro@libero.it

Il santuario di San Michele ad Olevano sul Tusciano in provincia di Salerno, fu uno dei maggiori santuari mete di pellegrinaggio dell'età carolingia. La natura impervia del luogo consente di ammirare ancora oggi quasi inalterate le architetture e le decorazioni altomedievali. Scavi archeologici realizzati tra il 2002 e il 2015 hanno permesso di apportare dati utili alla ricostruzione della vicenda del santuario e alla conoscenza della società longobardo-meridionale.

Keywords: Grotta di San Michele, Longobardi, Salerno, Monaco Bernardo

Il santuario di San Michele ad Olevano sul Tusciano, nel cuore del Mezzogiorno longobardo, costituisce un luogo privilegiato dove poter indagare le numerose problematiche legate ad un centro di pellegrinaggio internazionale dell'alto medioevo ${ }^{1}$. La malagevole accessibilità del sito e il declino della fortuna del santuario olevanese a partire dal XIII secolo, consentono ancora oggi di osservare quasi integro il luogo descritto dal monaco franco Bernardo che, di ritorno dalla Terrasanta, vi fece tappa. La grotta di San Michele si apre sulla costa settentrionale del monte Raione, il mons Aureus delle fonti altomedievali, nel territorio del comune di Olevano sul Tusciano (Sa), l'antico locus Tusciano, lungo la media-alta valle del Tusciano, un'importante direttrice naturale di collegamento tra le coste tirreniche e l'Adriatico, a circa $20 \mathrm{~km}$ da Salerno (fig. 1).

Il sito fa parte di un complesso monastico-santuariale che si struttura in due spazi funzionali distinti, uno interno alla grotta, il santuario vero e proprio, l'altro esterno. Alcune strutture difensive che si trovano a mezza costa e sbarrano $i$ sentieri che conducono alla grotta e i resti di un monastero poco distante dalla cavità, costituiscono la parte esterna finora nota del santuario. All'interno della grotta si snoda il percorso devozionale scandito da sei cappelle disseminate lungo un sentiero a partire da un'altura poco distante dalla bocca della grotta stessa fino alla più completa oscurità nel cuore della montagna (fig. 2).

Molto scarne risultano le notizie relative al santuario relativamente al periodo che qui si considera. La prima risale all'861, anno in cui l'Anonimo salernitano colloca la fuga da Salerno del vescovo eletto Pietro all'indomani della defe-

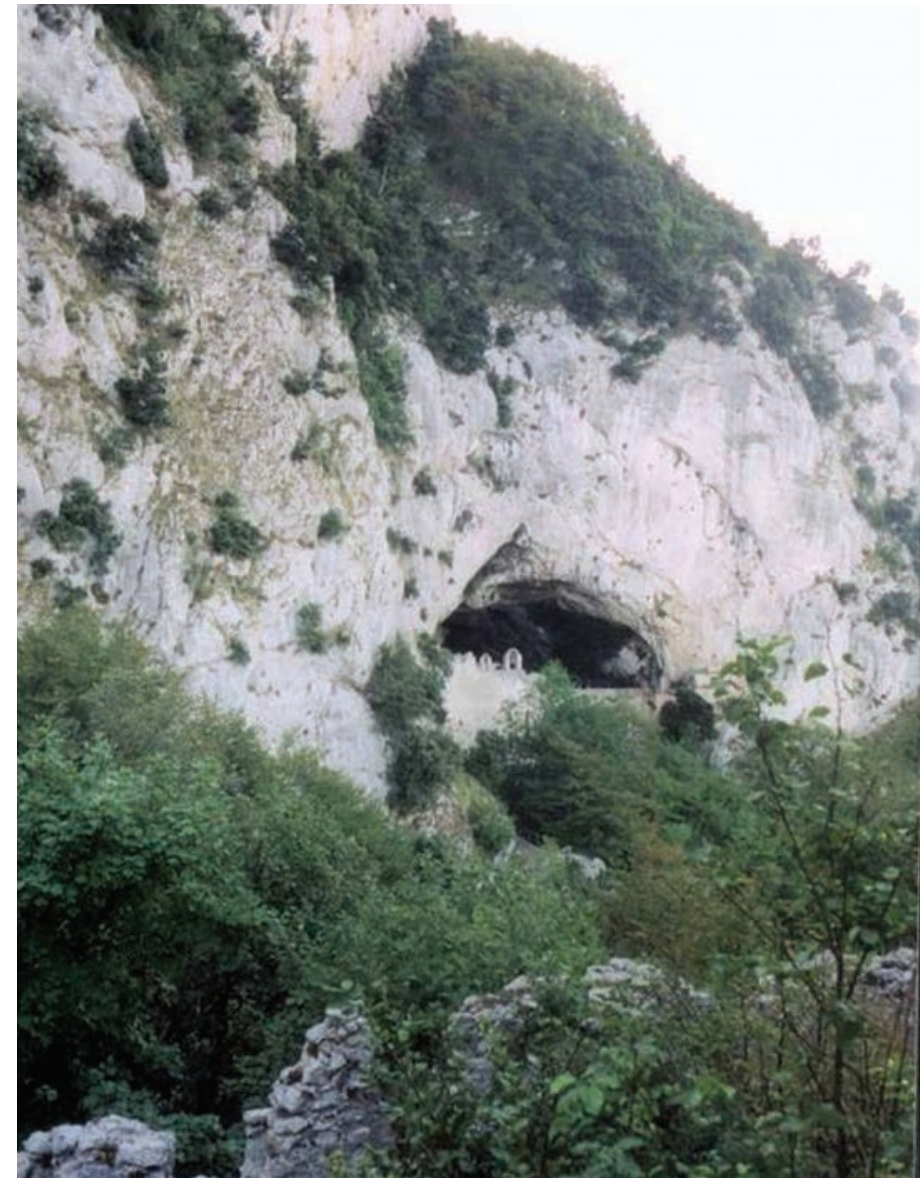

Fig. 1, Olevano sul Tusciano, Grotta di San Michele

\footnotetext{
${ }^{1}$ Si vedano almeno G. KALBY, La cripta eremitica di Olevano sul Tusciano, in Napoli Nobilissima, III, 1964; F. AVRIL, J.R. GABORIT, L'Itinerarium Bernardi monachi et les pélerinages d'Italie du Sud pendant le Haut-Moyen-Age, in Mélanges d'archéologie et d'histoire, LXXIX (1967); R. ZUCCARO, Gli affreschi nella Grotta di San Michele ad Olevano sul Tusciano, Roma, 1977; A. CAPODANNO, A. SALERNO, Scavi nella Grotta di San Michele ad Olevano sul Tusciano, in Archeologia medievale, XIX, 1992; A. Di Muro (a cura di), Luce dalla grotta: primi risultati delle indagini archeologiche presso il santuario di San Michele ad Olevano sul Tusciano, III Congresso della Società degli Archeologi Medievisti Italiani, Firenze, 2003; A. DI MURO - F. LA MANNA, Scavi presso la Grotta di San Michele ad Olevano sul Tusciano. Seconda relazione preliminare, in Archeologia medievale, XXXIII, 2006, p. 373-393; A. Di Muro (a cura di), La grotta di San Michele ad Olevano sul Tusciano. Storia, archeologia, arte di un santuario altomedievale, Olevano sul Tusciano, 2011.
} 


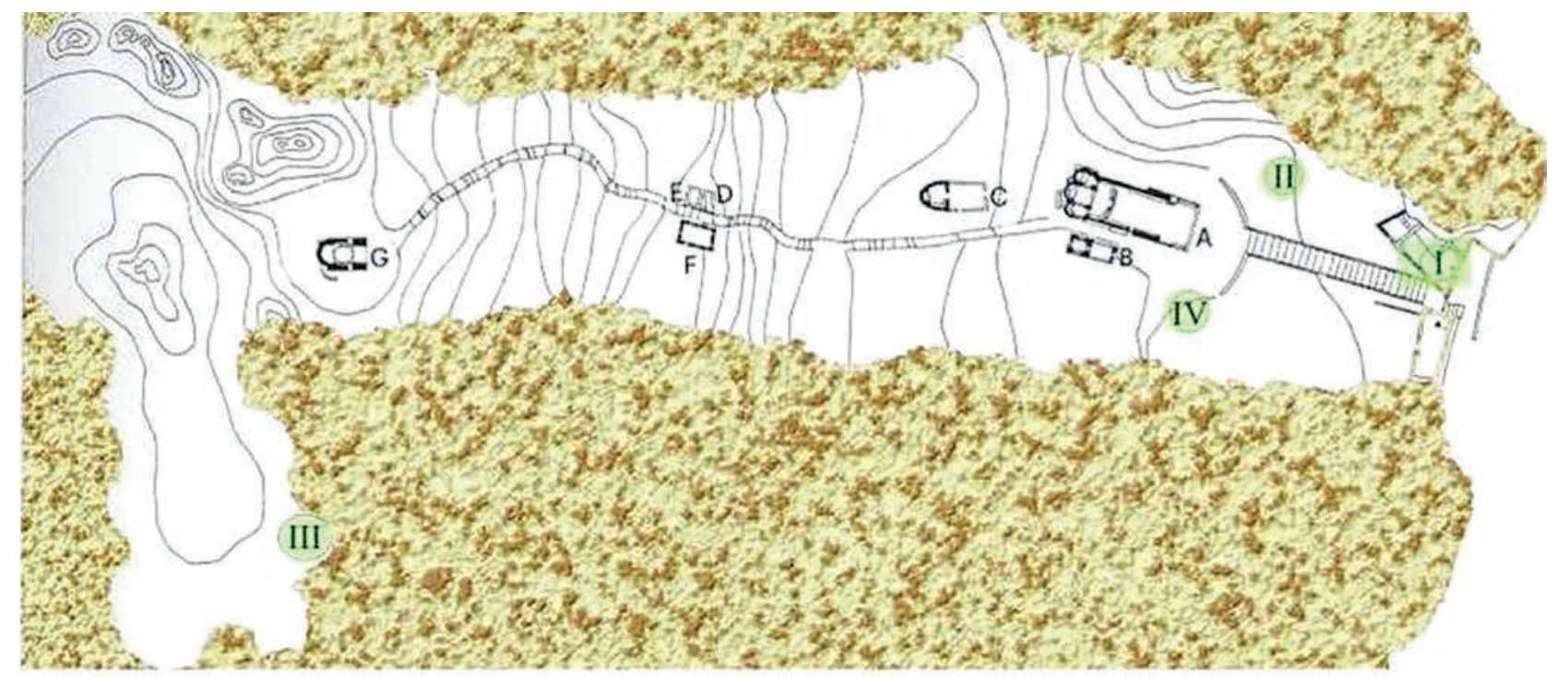

Fig. 2, Olevano sul Tusciano, Grotta di San Michele, Planimetria generale (modificata da Zuccaro 1977)

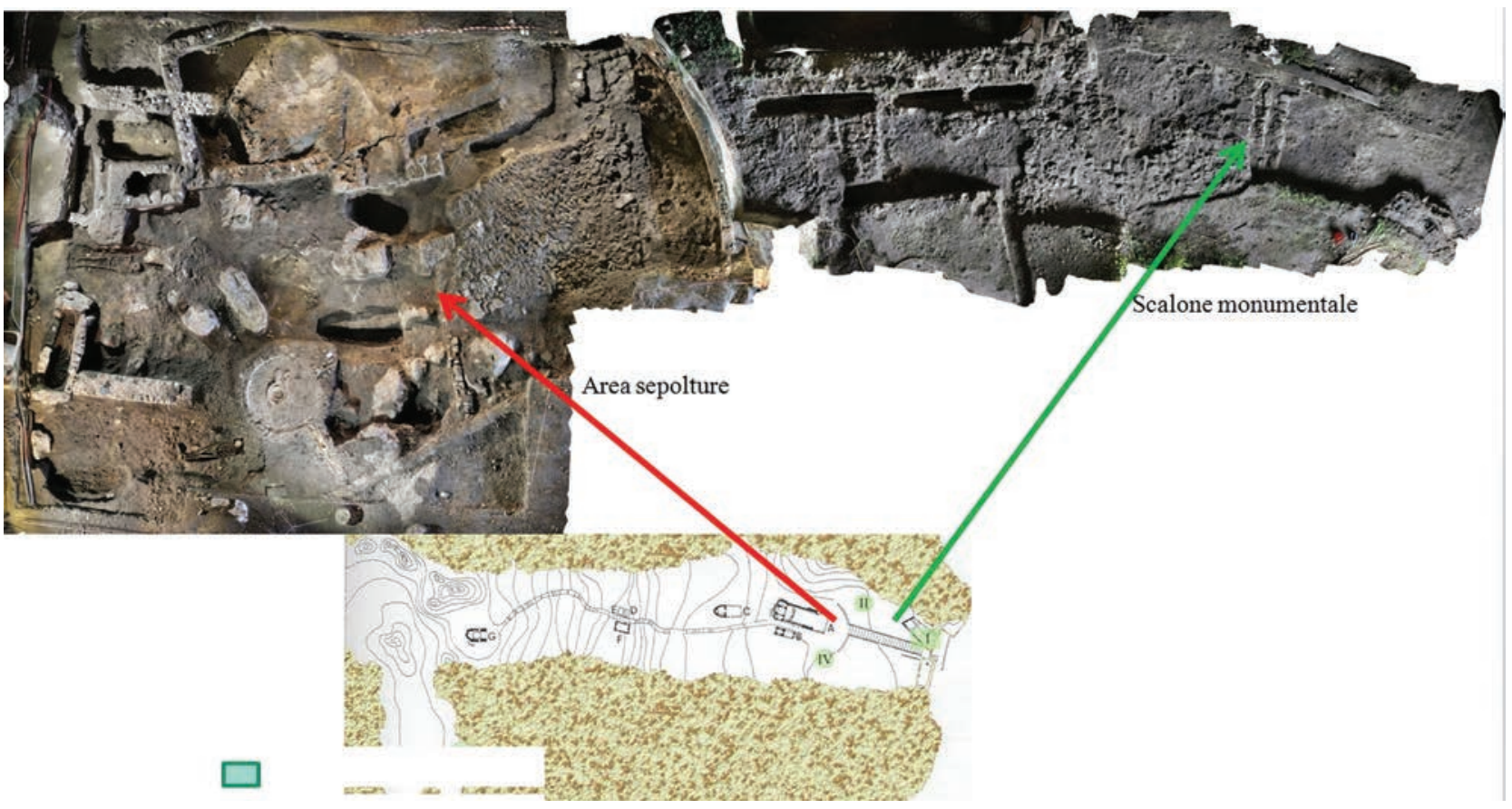

Fig.3, Olevano sul Tusciano, Grotta di San Michele, Area delle sepolture e scalone monumentale

nestrazione del padre, il principe Ademario: inseguito dal nuovo principe, Guaiferio e dal suo esercito, il presule trovò rifugio presso "Sanctum Angelum qui situm est in monte qui Aureus dicitur" ${ }^{2}$ dove rimase per un certo periodo. Nell'870 si ha la descrizione del monaco Bernardo, tratta dal resoconto del suo celebre pellegrinaggio. Questi, di ritorno dalla Terrasanta, sbarcato probabilmente a Salerno, fece visita al santuario del Tusciano, dove fu accolto dall'abate Valentino, ne descrisse sinteticamente la natura lussureggiante che lo circondava, l'oscurità dell'enorme caverna e i septem altaria che ne scandivano gli spazi ${ }^{3}$. L'inserimento negli itinerari di pellegrinaggio internazionale, testimoniato da Bernardo, dimostra il prestigio raggiunto dal santuario già in quegli anni. Le campagne archeologiche condotte all'interno e all'esterno del santuario a partire dal 2002 hanno permesso di ricostruirne, tra le altre cose, le strutture, potremmo dire, di 'servizio' e la qualità della cultura materiale di chi era preposto alla custodia.

La prima fase di frequentazione del santuario risale al VII secolo, testimoniata da alcune strutture riemerse nel corso delle indagini archeologiche e dalla costruzione dei bassi recinti sui quali si impostarono i sacelli ancora oggi visibili. Negli stessi anni, nell'area pianeggiante antistante l'attuale cappella dell'Angelo venne realizzato uno spazio funerario definito da due muri paralleli. Le sepolture erano individuate da pali ai quali erano legate catenelle di bronzo dalle quali pendevano lucerne vitree, pratica che si individua anche per le sepolture della fase successiva. In una seconda fase databile tra la seconda metà del VII secolo e la prima metà del successivo, si provvide ad ampliare lo spazio dedicato

\footnotetext{
${ }^{2}$ Chronicon Salernitanum, ed. U. Westerberg, A critical edition with studies on literary and historical sources and on language, in AUS, Studia latina Stockholmensia, Stockholm, 1956, p. 103, cap. 101.
}

3 F. AVRIL, J.R. GABORIT, op. cit. (n. 1), p. 318 ss. 


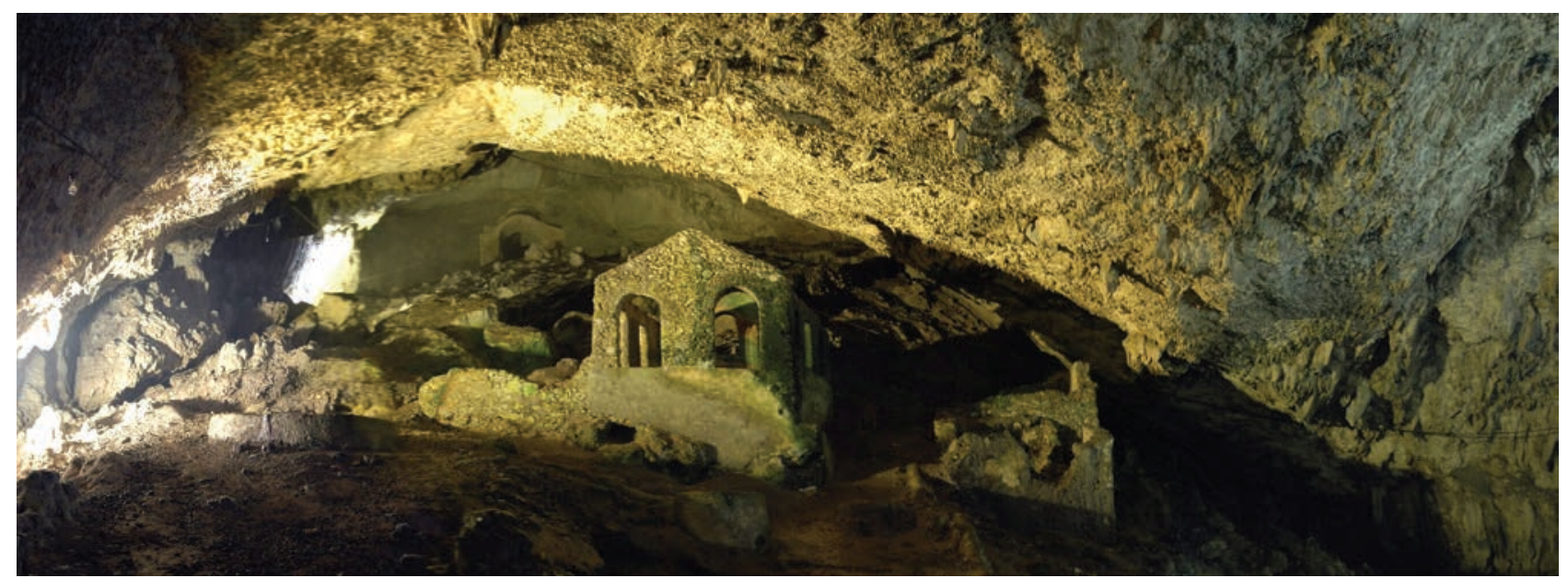

Fig. 4, Olevano sul Tusciano, Grotta di San Michele, Interno della Grotta.

alle sepolture, elevando altri muri che andarono a costituire un edificio di forma quadrangolare che, nel tempo, andò ampliandosi con la costruzione di un altro piccolo ambiente. L'ingresso avveniva da una sorta di protiro, individuato da quattro pilastri (fig. 3).

Tra la fine dell'VIII secolo e i primi anni del successivo si assiste ad una profonda riconsiderazione del santuario. All'ingresso della cavità si provvide all'elevazione di un edificio che si articolava su almeno due piani, probabilmente destinato all'ospitalità dei pellegrini. Al centro dell'imboccatura della grotta si realizzò in questo periodo uno scalone monumentale che, piegando verso occidente, si dirigeva, dopo aver lambito un grande edificio, verso il pianoro che ospitava le sepolture (fig. 3). La realizzazione dello scalone monumentale comportò alcune modifiche nell'area delle sepolture: il muro che delimitava verso nord l'edificio funerario venne rasato e in parte coperto dal selciato: tale azione non comportò tuttavia la defunzionalizzazione dello spazio riservato alle sepolture che continuarono ad essere realizzate in quello spazio.

A questa fase cronologica è possibile ricondurre un complesso intervento di riqualificazione dei sacelli del santuario che dall'originario basso recinto furono trasformate in piccole cappelle (fig. 4). Mi sembra interessante esaminare la prima cappella (fig. 5). Qui, su un preesistente piccolo recinto murario si innestò un edificio quadrangolare sormontato da una cupola conica, mentre sull'avancorpo si innalzò un parapetto in cui si ricavarono due nicchie decorate e rivestite da stucchi e sul timpano fu realizzato contestualmente un affresco raffigurante la Vergine Odighitria.

Le decorazioni in stucco realizzate nella cappella di Olevano trovano infatti interessanti confronti con la produzione scultorea "alta" di area longobarda tra l'VIII e il IX secolo. Di grande pregio risulta anche l'affresco che rappresenta la Madonna Odighitria, per il quale è possibile istituire confronti con alcuni affreschi di Santa Maria Antiqua al Palatino databili al tempo di Giovanni VII (705-707). L'apparato decorativo della cappella olevanese vale ad inserire il manufatto nella scia delle realizzazioni delle corti longobarde del Regnum e degli interventi di prestigio nella Langobardia minor (fig. 6).

Le altre cappelle, seppur meno appariscenti nelle decorazioni, mostrano una dinamica costruttiva analoga: ad un originario recinto basso, si giustappongono in una seconda fase strutture che conferiscono alle cappelle l'aspetto attuale. Una spazialità più complessa caratterizza l'ultima cappella del santuario olevanese (fig. 7). La struttura conserva una

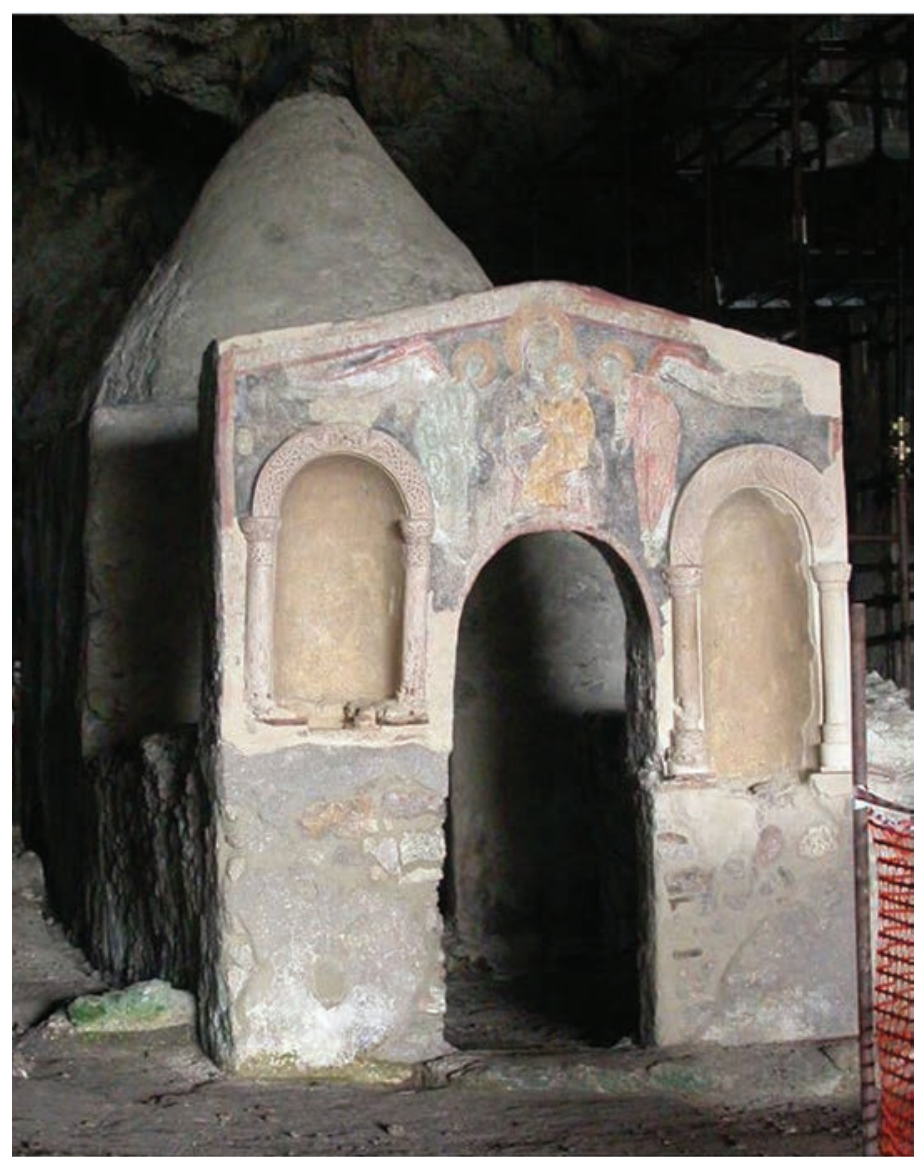

Fig. 5, Olevano sul Tusciano, Grotta di San Michele, La cappella Odighitria

fenestella confessionis, decorata con marmi policromi, cui si accedeva attraverso un deambulatorio ricavato dal primitivo recinto. Questa cappella, terminale di un percorso processionale ascendente ben preciso, doveva forse conservare reliquie oggetto di particolare venerazione come sembra attestare la gran profusione di iscrizioni graffite sugli intonaci delle pareti interne. Il rinvenimento al di sotto della fenestella di un deposito di pietre forate interpretabili come segni penitenziali dei pellegrini, rappresenta un'ulteriore riprova del carattere centrale dell'ultima cappella nell'ambito del santuario olevanese.

Una volta giunti all'ultimo sacello, i pellegrini dovevano continuare il loro cammino fino alla piccola vasca realizzata nel braccio orientale della grotta. Si tratta di un manufatto che raccoglie l'acqua stillante dalla volta della grotta, affiancato da una stalagmite sapientemente lavorata in modo da 


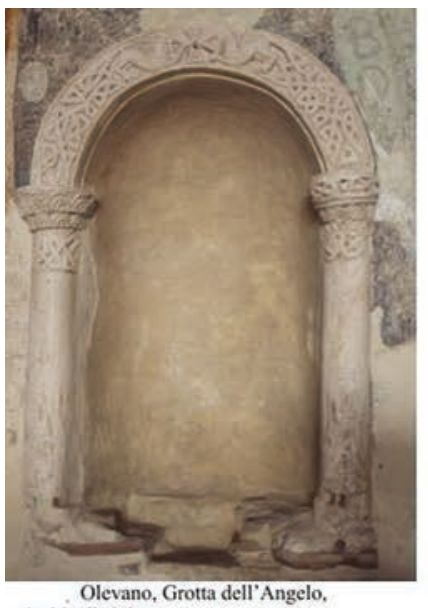

nicchia di sinistra

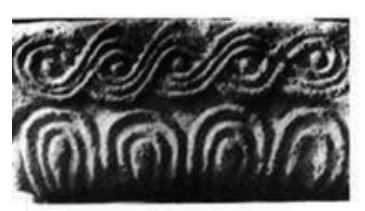

Brescia, Santa Maria di Gussago, iequasantiera (da Panazza-Tagliaferri 1966)

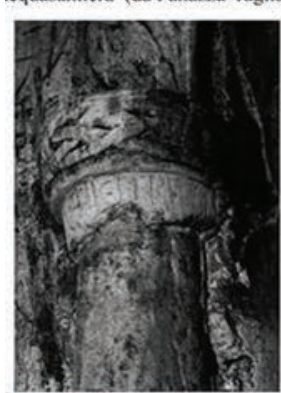

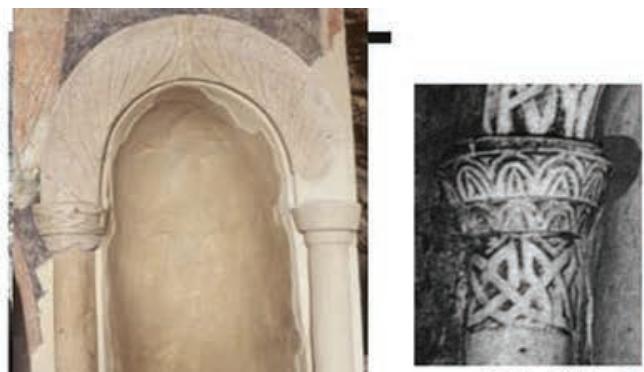

, Grotta dell'Angelo

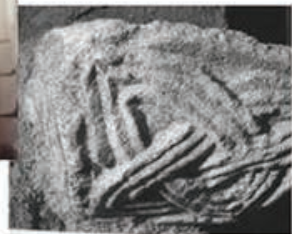

Santa Cristina, capitello

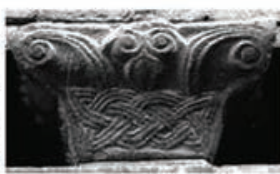

Milano, S. Vincenzo, ca (da Romanini 1992)

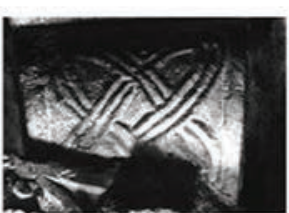

Milano, Campanil

der Monaci, capitello
(da Romanini 1992

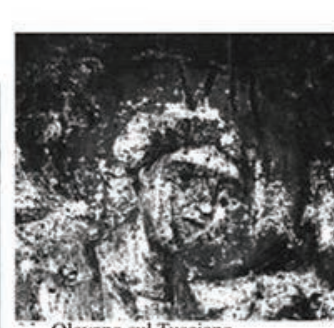

Olevano sul Tusciano

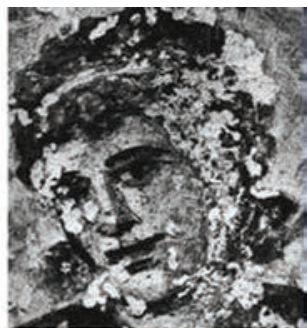

Santa Maria Antiqua
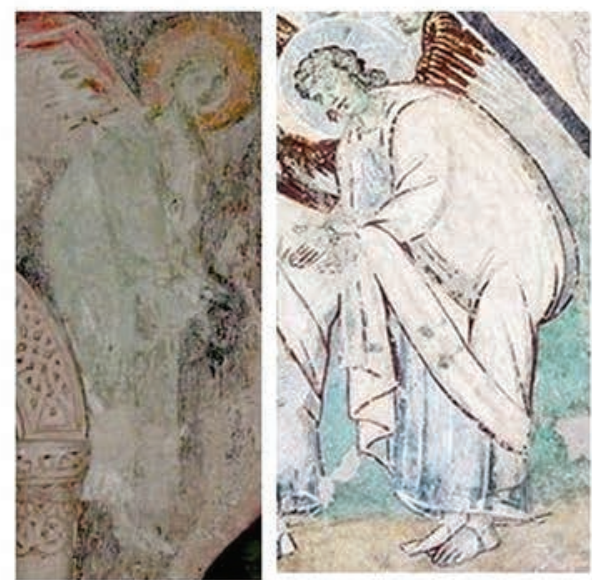

Olevano, Grotta dell'Angelo. capitello con iscrizione

Fig. 6, Olevano sul Tusciano, Grotta di San Michele, Decorazioni della cappella Odighitria e confronti

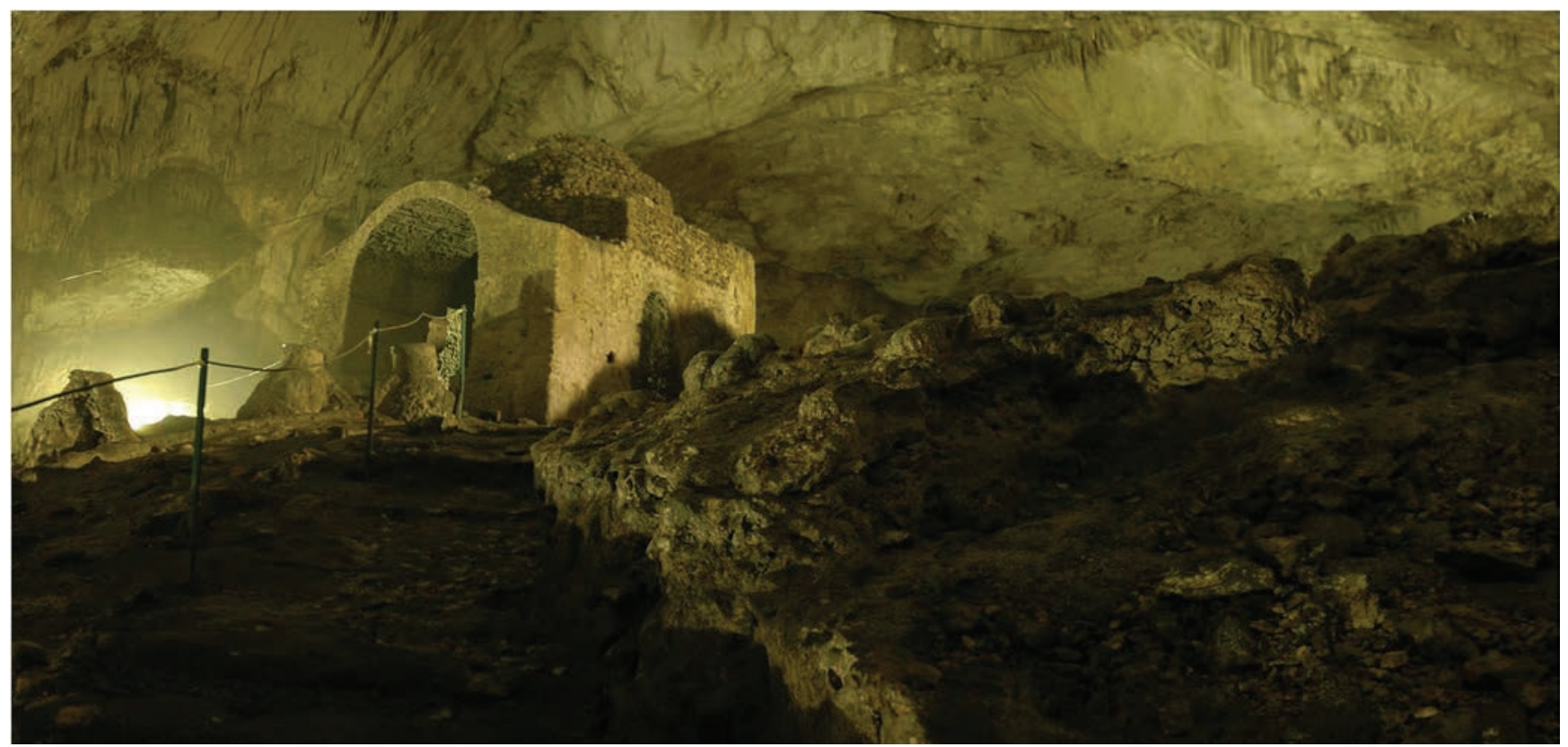

Fig. 7, Olevano sul Tusciano, Grotta di San Michele, ultima cappella

realizzare un fusto di colonna (fig. 8). Non appare inverosimile che, sul modello garganico, si considerasse l'acqua raccolta nella vasca apportatrice di benefici effetti per i pellegrini che ne avessero bevuto a conclusione del viaggio all'interno del profondo antro olevanese, come testimoniano alcuni documenti del XVIII secolo4.

${ }^{4}$ Liber de apparitione Sancti Michaelis in monte Gargano, ed. G. Waitz, in Monumenta Germaniae Historica, Scriptores rerum Langobardicarum et Italicarum, Hannover, 1878, p. p. 542; M. TROTTA, I luoghi del Liber de Apparitione, in C. Carletti, G. Otranto (a cura di), Culto e insediamenti micaelici nell'Italia meridionale fra tarda antichità e Medioevo, Bari, 1994, p. 130-133. 


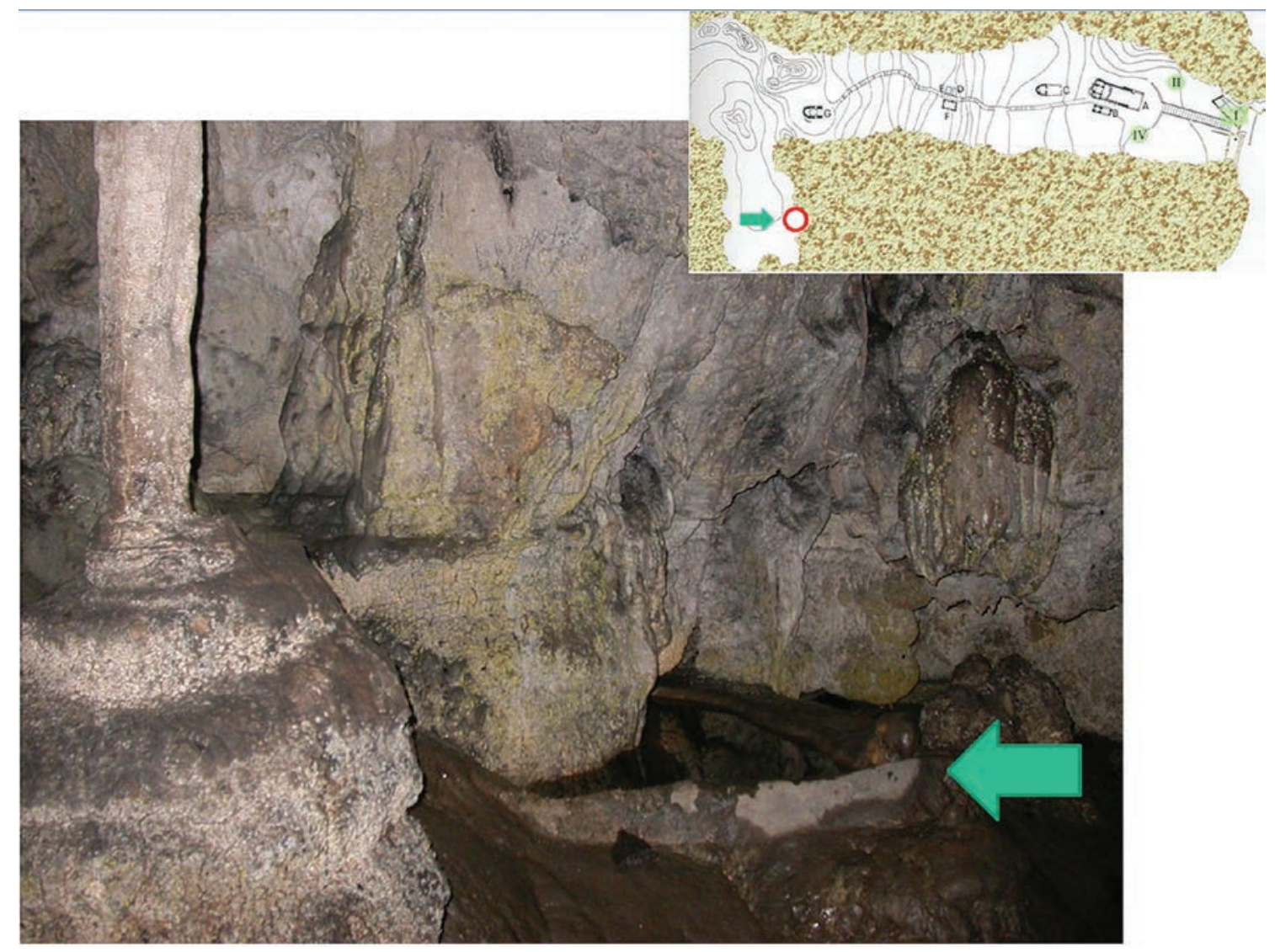

Fig. 8, Olevano sul Tusciano, Grotta di San Michele, vasca

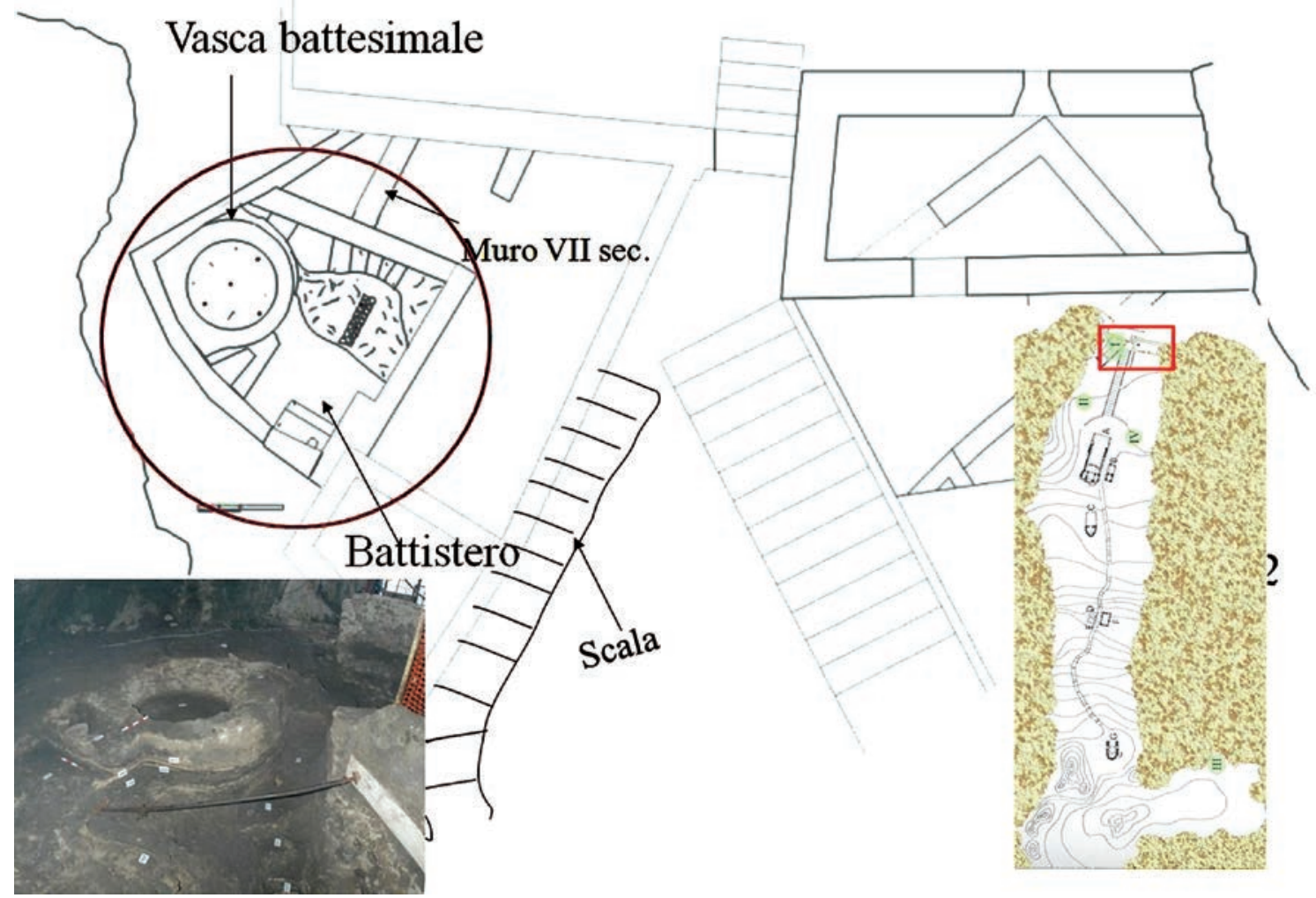

Fig. 9, Olevano sul Tusciano, Grotta di San Michele, battistero e strutture all'ingresso

Nella seconda metà IX, nei pressi dell'antico muro del santuario, si elevò un piccolo edificio battesimale, forse in connessione con la già ricordata presenza del vescovo salernitano Pietro. L'aula battesimale presenta una pianta quadrangolare e risulta interamente intonacata. Nella parte settentrionale del vano si realizzò la vasca battesimale circolare, intonacata all'interno e all'esterno. Quattro fori al centro della vasca permettevano il fluire dell'acqua che veniva convogliata in un canale coperto da una voltina a botte (fig. 9).

Le indagini hanno riportato alla luce oltre 50000 reperti databili tra VII e XII secolo: reperti ceramici, archeozoo- 


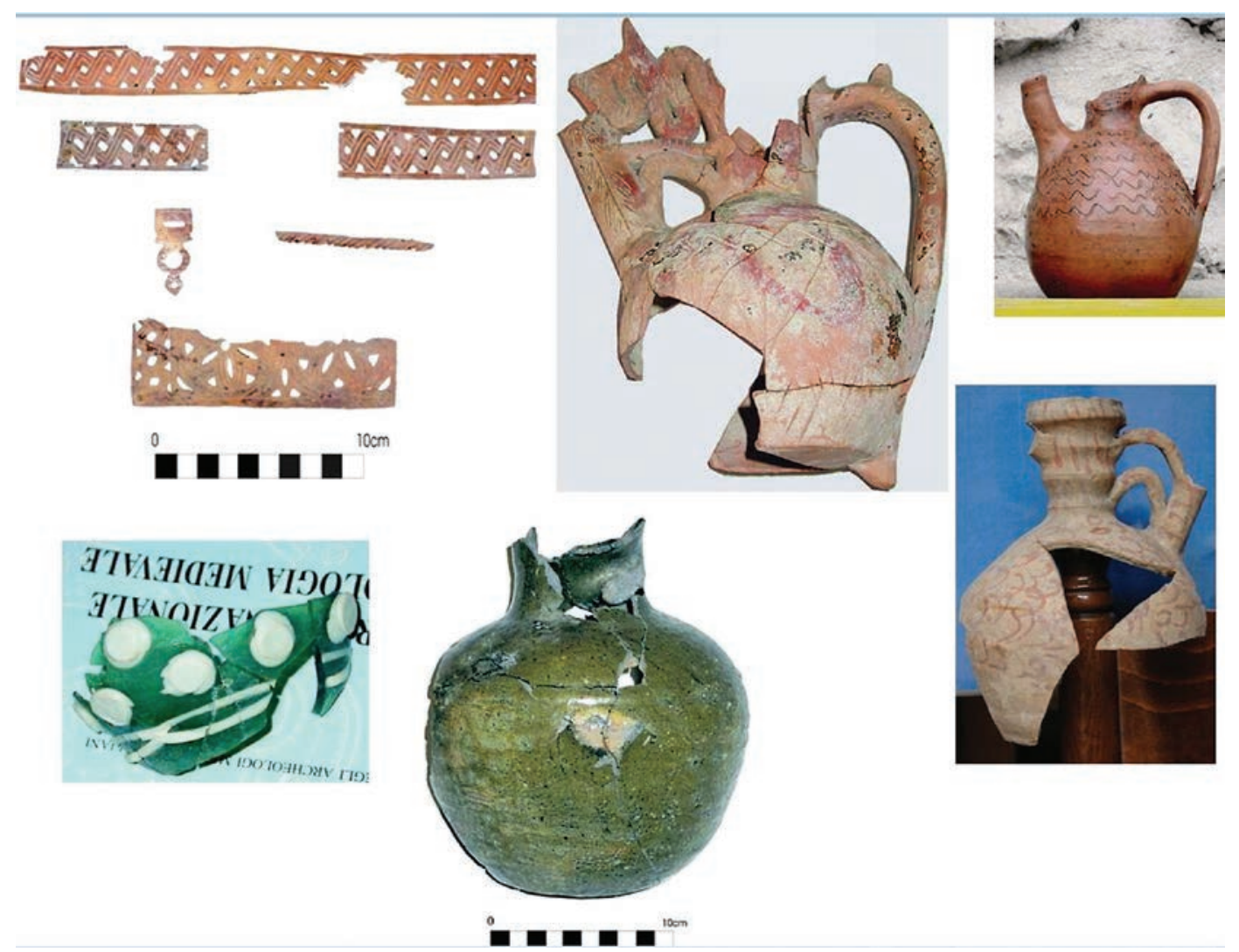

Fig. 10, Olevano sul Tusciano, Grotta di San Michele, alcuni reperti provenienti dagli scavi

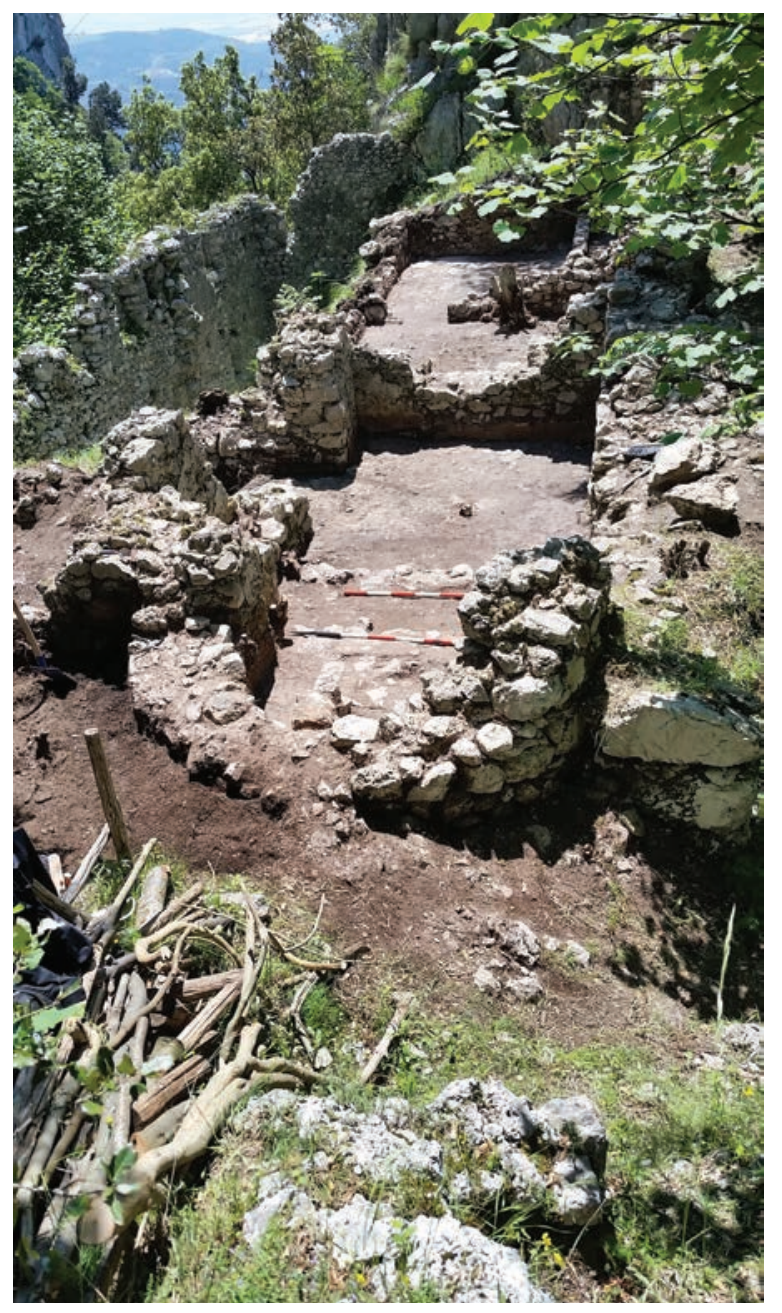

Fig. 11, Olevano sul Tusciano, monastero all'esterno della Grotta logici, vitrei, metallici, ossei, architettonici, costituiscono un immenso serbatoio di informazioni, ancora in parte da analizzare compiutamente, che getta luce rinnovata sulle vicende della grotta di San Michele (fig. 10). Si tratta talvolta di oggetti preziosi: coppe vitree con decorazioni, speroni dorati (probabilmente ex voto), sovraccoperte di evangelari, ma forse è la ceramica a fornire la misura più immediata del livello della cultura materiale di chi abitava e frequentava questo luogo straordinario.

Il materiale ceramico rinvenuto (oltre 40000 frammenti databili tra VII e XI secolo), si inserisce per lo più nell'alveo delle produzioni locali (anche se non mancano oggetti provenienti da lontano, dall'Iraq, dall'Africa del Nord, dalla Sicilia, da Roma), ma si segnala spesso per la qualità inusitata delle realizzazioni. Dagli scavi provengono, ad esempio, numerosi acquamanili datati a partire dal IX secolo, il cui uso è probabilmente da mettere in relazione con le funzioni liturgiche che si celebravano nel santuario, in particolare con le celebrazioni battesimali. Di particolare interesse in questo senso risulta la splendida brocchetta acquamanile con decorazione plastica applicata rappresentante forse Giona rigurgitato dal Pistrice, figura del Risorto e della rigenerazione battesimale del neofita: un pezzo unico nel panorama delle produzioni altomedievali, paradigmatico delle possibilità tecnico-decorative di un figulo della Langobardia minor ${ }^{5}$.

La raffinatezza e il pregio degli oggetti cui si è accennato, valgono a qualificare il rango dei componenti la comunità monastica, soprattutto per la fase altomedievale, ma anche gli oggetti ceramici di uso quotidiano (brocchette, ceramiche da fuoco, ceramica a vetrina pesante) mostrano una qualità che indica una condizione sociale decisamente elevata di chi ne faceva uso (ad esempio è singolare il rinvenimento di un contenitore di medie dimensioni interamente decorato

5 P. SAPORITO, La ceramica, in Luce dalla grotta, op. cit. (n. 1), p. 400-403. 
con bande rosse incise: doveva forse accogliere un liquido particolarmente pregiato $)^{6}$.

Un altro indicatore di grande interesse per comprendere la vita quotidiana che si svolgeva presso il santuario olevanese, è fornito dall'analisi dei residui alimentari osteologici rinvenuti nella discarica. Si tratta di reperti relativi a capriovini, bovini, suini, che mostrano l'incidenza dell'allevamento nelle abitudini alimentari di chi frequentava quei luoghi. Il rinvenimento di teschi di bovini e di capriovini, oltre che di altre specie, in particolare di suini, induce a ritenere che la macellazione e l'allevamento di alcuni animali, avvenisse in loco, circostanza che fornisce interessanti suggestioni sull'organizzazione del monastero, cui forse si affiancava una o più famiglie di servitori ${ }^{7}$.

Alla luce delle analisi condotte sui reperti archeozoologhici si può presumere che i monaci fornissero ai pellegrini che si recavano sul mons Aureus il pasto, come era nella tradizione dell'accoglienza monastica e, più in particolare, in connessione con i grandi santuari. Possiamo immaginare che i viandanti si accontentassero delle parti meno nobili degli animali macellati, bollite, come si desume dalle analisi, nelle numerose olle da cucina rinvenute nei medesimi strati forse usate per insaporire zuppe e minestre (di cui sono stati rinvenuti resti), mentre i monaci si può presumere consumassero le parti più pregiate. Un menu peraltro adeguato alle necessità nutrizionali di chi era giunto al santuario al termine un faticoso cammino. Probabilmente allo stesso Bernardo e ai suoi compagni, pellegrini al santuario negli anni di attività della discarica, fu offerto un pasto di questo tipo alla tavola dell'abate Valentino.

L'ipotesi di una sorta di hospitium in prossimità dell'apertura della grotta potrebbe trovare elementi di conferma nella natura impervia del complesso olevanese; l'unica area all'esterno della grotta in cui si poteva costruire un edificio per l'ospitalità dei pellegrini era stata utilizzata per elevare il monastero, riportato alla luce da una campagna di scavi (fig. 11) e le cui prime fasi sono riferibili alla fine dell'VIII secolo, per cui gli spazi disponibili si riducevano all'interno del santuario, nei pressi dell'antica scala d'accesso ai sacelli, situazione, tra l'altro, che trova un parallelo nella longa porticus del sacrario micaelico del Gargano.

Alla fine del X secolo con la donazione all'arcivescovo di Salerno segnata dalla costruzione della cappella dell'Angelo, iniziò il declino del santuario (figg. 12, 13). Un santuario

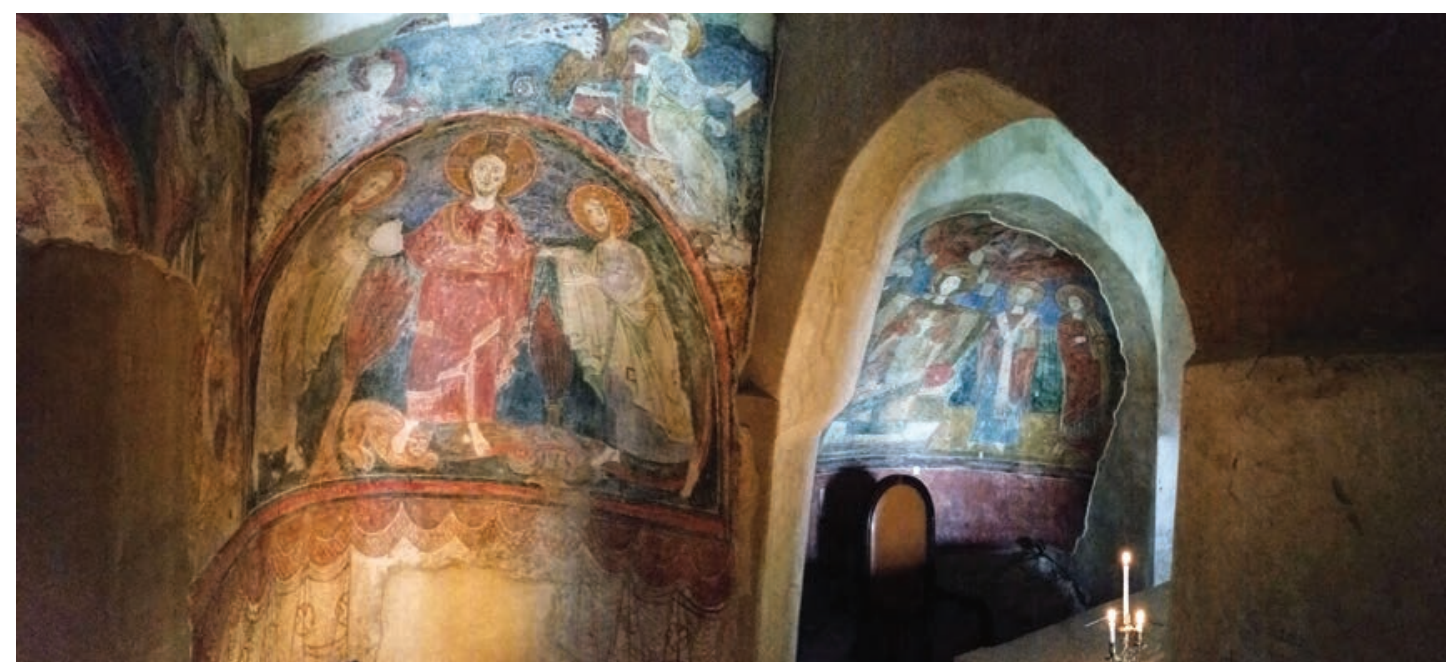

Fig. 12, Olevano sul Tusciano, Grotta di San Michele, La Cappella dell'Angelo

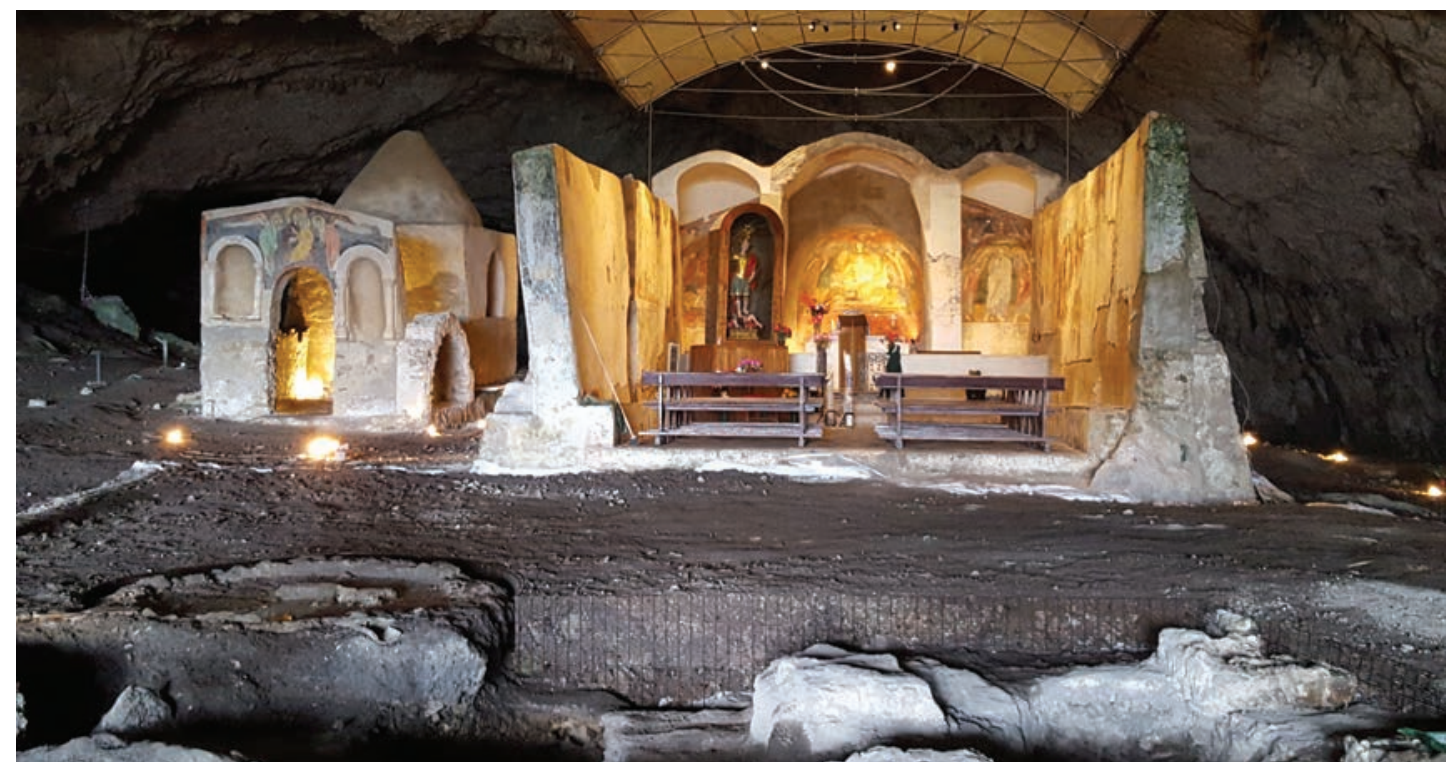

Fig. 13, Olevano sul Tusciano, Grotta di San Michele, La Cappella dell'Angelo, Affreschi absidali.

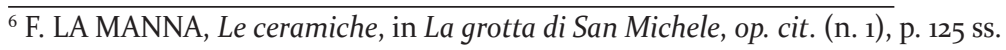

${ }^{7}$ M. MASTRANGELO, Le ceramiche da fuoco, in La grotta di San Michele, op. cit. (n. 1), p. 163 ss. F. LA MANNA, L'indagine archeozoologica, in La grotta di San Michele, op. cit. (n. 1), p. 205 ss. 
quello del mons Aureus che rivela una complessità organizzativa notevole, che andrà meglio precisata con nuove indagini, ma che si può sinteticamente così definire: un monastero poco lontano dall'imboccatura della grotta, cui afferisce un nucleo di servitori dimoranti forse all'interno di un ampio recinto murario individuato in seguito ad alcune ricognizioni lungo il versante nord orientale del monte, il cui compito è legato essenzialmente all'allevamento delle mandrie del cenobio (bovini, capriovini, maiali, pollame) e forse alla coltivazione delle terre circostanti.

I monaci, come si deduce anche dall'accenno che ne fa Bernardo, sono preposti alla vigilanza del santuario e all'accoglienza dei pellegrini i quali possono trovare un pasto caldo e ospitalità nei locali all'interno della grotta. L'edificio battesimale valse a conferire un prestigio ulteriore al santuario. Un piccolo cimitero sul pianoro tra l'imboccatura della grotta e la cappella decorata con stucchi costituiva il luogo dell'ultima dimora di quei monaci che avevano dedicato la loro esistenza a custodire il prestigio del santuario olevanese ma anche degli abitanti dei villaggi circostanti o di benefattori laici, come si evince dal rinvenimento di inumati di genere femminile (almeno $4 \mathrm{su} 21$ sepolture esplorate) oltre che di bambini. Una lucerna di vetro pendente da un palo indicava l'ultima dimora di questi devoti dello psicopompo.

Il quadro, peraltro ancora largamente incompleto, che scaturisce dalle tracce finora disponibili, mostra caratteri in parte comuni all'organizzazione di coeve comunità monastiche quali San Vincenzo al Volturno e Montecassino o con luoghi di culto quali il santuario garganico ${ }^{8}$, sebbene il santuario olevanese si differenzi da quest'ultimo per alcuni aspetti sostanziali quali, ad esempio, la caratteristica polidevozionale riscontrabile ad Olevano, evidente nelle 5 cappelle dotate di altari all'interno dei quali erano conservate le reliquie dei santi venerati, elemento assente nel santuario di Monte Sant'Angelo.

\footnotetext{
${ }^{8}$ Per San Vincenzo al Volturno si vedano almeno R. HODGES, In the shadows of Pirenne: San Vincenzo al Volturno and the revival of Mediterranean commerce, in R. Francovich, G. Noye (a cura di), La storia dell'alto Medioevo italiano alla luce dell'archeologia, Siena, 1994, p. 109, R. HODGES, Light in the Dark ages. The rise and fall of San Vincenzo al Volturno, London, 1997; F. Marazzi (a cura di), La "Basilica Maior" di San Vincenzo al Volturno (scavi 2000 - 2007), Cerro al Volturno, 2014, con bibliografia.
} 